\title{
Dynamic material strength measurement utilizing magnetically applied pressure-shear
}

\section{C.S. Alexander ${ }^{\mathrm{a}}$}

Sandia National Laboratories, Albuquerque, NM 87185-1195, USA

\begin{abstract}
Magnetically applied pressure-shear (MAPS) is a recently developed technique used to measure dynamic material strength developed at Sandia National Laboratories utilizing magneto-hydrodynamic (MHD) drive pulsed power systems. MHD drive platforms generate high pressures by passing a large current through a pair of parallel plate conductors which, in essence, form a single turn magnet coil. Lorentz forces resulting from the interaction of the self-generated magnetic field and the drive current repel the plates and result in a high pressure ramp wave propagating in the conductors. This is the principle by which the Sandia Z Machine operates for dynamic material testing. MAPS relies on the addition of a second, external magnetic field applied orthogonally to both the drive current and the self-generated magnetic field. The interaction of the drive current and this external field results in a shear wave being induced directly in the conductors. Thus both longitudinal and shear stresses are generated. These stresses are coupled to a sample material of interest where shear strength is probed by determining the maximum transmissible shear stress in the state defined by the longitudinal compression. Both longitudinal and transverse velocities are measured via a specialized velocity interferometer system for any reflector (VISAR). Pressure and shear strength of the sample are calculated directly from the VISAR data. Results of tests on several materials at modest pressures ( $10 \mathrm{GPa})$ will be presented and discussed.
\end{abstract}

\footnotetext{
a e-mail: calexa@sandia.gov
}

This is an Open Access article distributed under the terms of the Creative Commons Attribution License 2.0, which permits unrestricted use, distribution, and reproduction in any medium, provided the original work is properly cited. 